\title{
Consensus development conference: coronary artery bypass grafting
}

Britain's first consensus development conference was held in London from 21 to 23 November, sponsored by the King's Fund in association with the Royal College of Physicians and the Royal College of Surgeons. A panel of doctors and other professionals, including lawyers and economists, listened to expert evidence and debated it first in public and later in closed session. The panel's report appears below (see leading article at $p 1477$

\section{Consensus statement}

Coronary artery disease is one of the major health problems in the Western world today. Coronary artery bypass surgery is a technique in which a blocked or narrowed section of a coronary artery is bypassed using part of a vein or artery from elsewhere in the patient's body. The treatment has two separate objectives: the relief of angina and the prolongation of life. There are, however, alternative methods of treatment for coronary artery disease, and there are a wide variety of views about which patients stand to benefit from coronary artery bypass surgery, especially in comparison with these alternatives.

Britain shares with Sweden, France, and Germany a rate of bypass operations per million population that is about one sixth of that in the United States, a quarter of that in Australia, and a third of that in Holland. Within the United Kingdom the rates over the five vear period 1977-82 varied 10-fold among regions. These variations reflect differences in the availability of facilities for investigation and surgery and probably also the differing views of doctors and patients about the indications for the procedure. A major question is whether there should be any change in the number of coronary artery bypass operations over the next few years, taking into account other demands on health care resources. Coronary artery bypass surgery is only one aspect, albeit an important one, of the whole massive problem of coronary artery disease.

The King's Fund in association with the Royal College of Physicians and Royal College of Surgeons of England sponsored this first consensus development conference on the subject of coronary artery bypass surgery in an effort to resolve some of the questions about this procedure. For one and a half days a 12 member panel listened to evidence from experts and from the participants at the open conference. They then prepared answers to four questions which had been set in advance.

(1) What are the pros and cons of coronary artery bypass surgery (compared with alternatives) for various types of patient (including age and sex), in terms of survival and quality of life?

It is possible to list advantages and disadvantages of medical and surgical treatment which apply, to some extent, to all patients with coronary artery disease:

(1) For the relief of angina surgery is effective in most cases where medical treatment including drug therapy and modification of lifestyle are ineffective or unacceptable.

(2) $\beta$ blocking drugs in particular, even when relieving angina,

The panel comprised: Professor Bryan Jennett, Mr John Dark, Professor Gerald Dworkin, Dr Geoff Watts, Dr Malcolm Forsythe, Dr Anthony Wing, Ms Oriole Goldsmith, Professor Michael Healy, Ms Angela Heslop, Professor John Goodwin, Ms Anne Ludbrook, Dr Raanan Gillon.

Invited experts presenting evidence were: Professor Michael Oliver, Dr Celia Oakley, Dr Brian Maurer, Professor Michael R Bond, Mr John Parker, Professor John Hampton, Ms Elizabeth Yates, Dr Michael C Petch, Dr D N S Kerr, Professor J Wheatley, Sir Keith Ross, Dr David De Bono, Dr Douglas Chamberlain, Professor Alan Williams. are liable to produce various side effects and a general lowering of vitality. Surgery on the other hand is often followed by an improvement in well being.

(3) Medical treatment can be implemented immediately following diagnosis. Surgery under present conditions is liable to involve considerable delay, which will add to the patient's anxiety.

(4) Surgery must be preceded by costly and arduous investigations. These can be avoided with medical treatment but at the price of the detailed pathology remaining unidentified.

(5) The immediate financial costs of surgery are considerably higher than those of drug treatment. In the medium to long term the balance of cost is less certain; medical cases require closer medical supervision in hospital and in the community. Either treatment may become ineffective so that further treatment (possibly surgical) may have to be undertaken. The full benefits of surgery require counselling and rehabilitation measures, and these are not always provided.

(6) Surgical cases are subject to a small operative mortality (1\% is being achieved in some centres; the UK average in 1982 was $3 \cdot 2 \%)$. There is postoperative pain and discomfort and also a liability to complications. All open heart surgery may be followed by adverse psychological effects, and temporary neurological changes have been reported.

As far as specific groups are concerned women, who form around $10 \%$ of all cases, have a higher operative mortality than men. Older patients in general have a higher operative mortality and morbidity and are more likely to be able and willing to alleviate symptoms by reducing activity, though increasing numbers of older patients are receiving surgery with beneficial effect. On the other hand, complete symptom relief may be more important to younger men and women with work and family responsibilities.

It is important to re-emphasise that treatment has two separate objectives: relief of angina and prolongation of life. For relief of angina surgery succeeds in many cases where drugs fail and avoids their side effects.

For survival the situation is more complex. In anatomically severe (left main stem) disease surgery is widely agreed to prolong life. With less severe forms, the evidence mainly comes from the European Coronary Surgery Study and Coronary Artery Surgery Study trials. These are not in full agreement but are consistent with a somewhat better five year survival with surgical rather than medical treatment in patients with three vessel disease. Less severe cases (one vessel disease) have a good prognosis without surgery. It must be remembered that both trials excluded as not randomisable cases with severe angina and used the medical and surgical methods of the last decade. More refined classification, now possible, can lead to better decisions about treatment.

\section{SUGGESTIONS FOR CLINICAL PRACTICE}

For the quantitative discussions below, it is necessary to have an explicit pattern of investigation and treatment. In the light of what we have heard, we make the following suggestions for good clinical practice (irrespective of resources):

(1) Patients with stable angina (fig 1) are given immediate medical treatment. If this is effective there is a case for further investigation by exercise (stress) testing (and echocardiography where available). If these tests are judged positive at a low work load angiography follows. Severe cases (left main stem and triple vessel disease) receive coronary artery bypass surgery; mild cases (two vessel and single vessel disease) receive either coronary artery bypass surgery or angioplasty. Patients whose medical treatment is unsuccessful require angiography without preliminary exercise testing, as do those whose angina is unstable. 
(2) Asymptomatic patients who survive a myocardial infarct (myocardial infarction) (fig 2 ) may receive $\beta$ blocking drugs and may require exercise tests. Those with positive tests require angiography and surgery as above.

(2) What are the indications for various investigations for coronary artery disease?

Investigations should usually be limited to implementing the management pattern suggested above. The first and most crucial investigation is thorough clinical assessment based on a good medical history and examination. Angina which is controlled medically should be investigated with chest $x$ ray examinations, resting electrocardiography, and exercise (stress) electrocardiography under cardiological supervision based on an accepted protocol. Echocardiography may be helpful. Significant and relevant cardiac abnormalities should be followed up with cardiac angiography (with or without supplementary radionuclide investigation) with a view to determining suitability for operation.

Another category of patients who may need investigation in this way, because a proportion of them may be suitable candidates for coronary artery bypass surgery, comprises patients who have had myocardial infarction but who do not have angina. Angina which is not controlled medically should be investigated by cardiac angiography. Again, additional radionuclide investigations may be helpful in elucidating remaining uncertainties.

Coronary artery angiography in a regional centre is generally considered to be the definitive investigation for accurate diagnosis of coronary artery pathology and estimation of left ventricular function and is necessary in all cases being considered for coronary artery bypass surgery. However, it is expensive and carries a small risk of mortality and morbidity. For these reasons and the convenience of patients we recommend a preliminary assessment in the district general hospital using non-invasive exercise electrocardiographic testing. This technique, when carried out according to an acceptable protocol, will identify most patients who might benefit from coronary artery bypass surgery. Echocardiography is recommended both for its assistance in determining left ventricular function and to elucidate other cardiac, notably valvular, pathology.

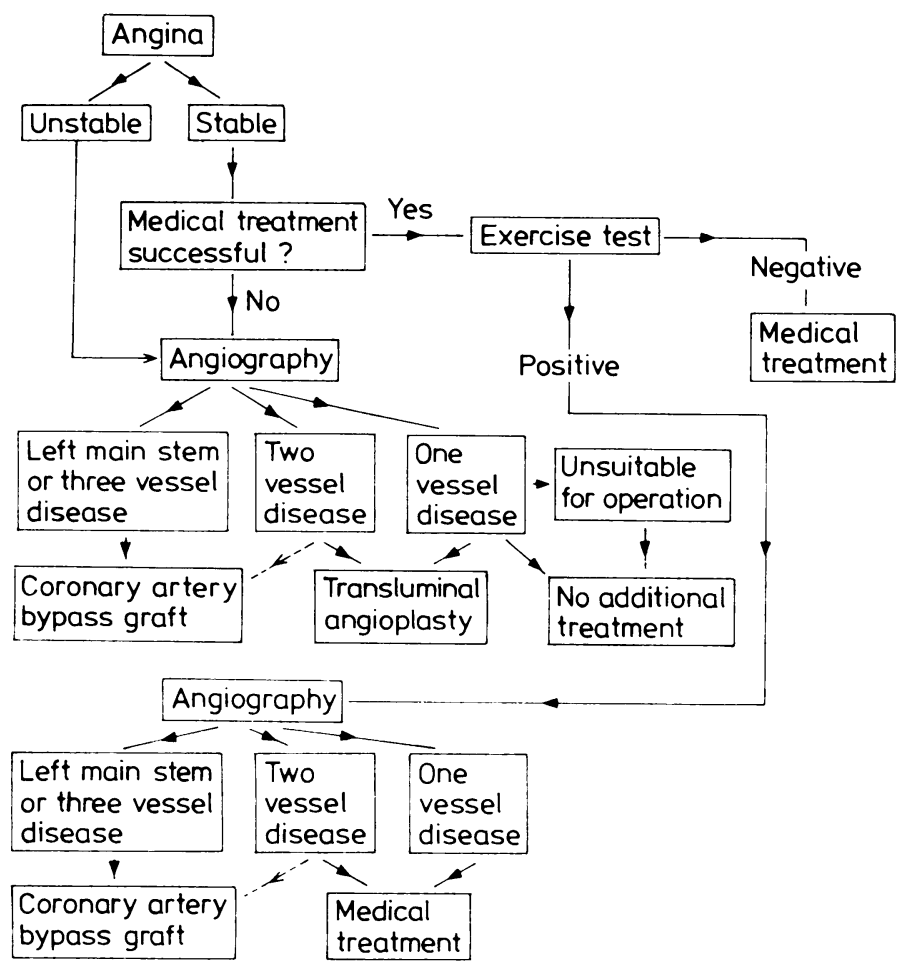

FIG 1--Suggested assessment and treatment for patients with angina.

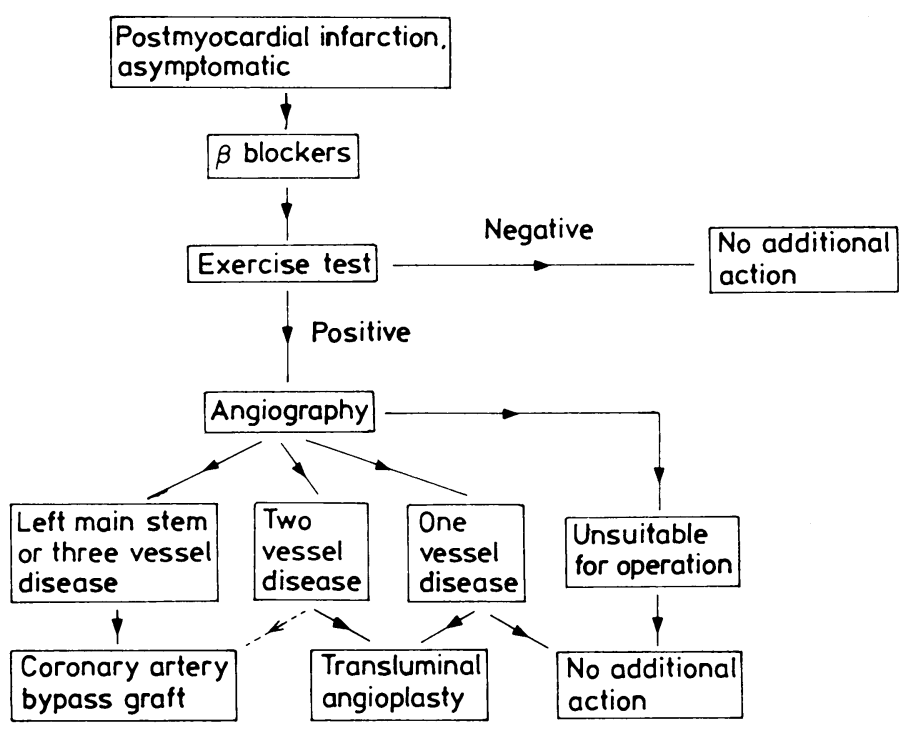

FIG 2-Suggested assessment and treatment for asymptomatic patients who survive a myocardial infarction.

(3) What size are the potential pools of patients for investigations and for coronary artery bypass surgery, taking account of alternative therapies? Are these estimates likely to change substantially in the next five to 10 years?

The panel has considered numerous estimates of the potential coronary artery bypass surgery workload, based on international activity levels and limited UK data on the incidence and prevalence of angina and myocardial infarction. There are three types of patients: (1) New angina patients with an annual incidence of between 110 and 140/million crude population with characteristics making them suitable for surgery. (2) A sizable backlog of patients with chronic angina, which will undoubtedly vary because of the gross geographical differences in the level of service provision. (3) Patients who have survived recent myocardial infarction. Of these, about a third develop angina which is in addition to the estimate of new angina patients. Those without angina may have patterns of vessel occlusion that make them at high risk of recurrence of myocardial infarction. The exact number of these cases is uncertain, but applying American workload figures to the sparse UK data gives an annual range of between 200 and 550 cases of coronary artery bypass surgery/million population.

However, there appears to be no information nationally available about the distribution of these three types of cases in the current coronary artery bypass surgery throughput in the UK, nor are there data on the severity categories of these patients. Nevertheless, the above categories produce an annual range of between 300 and 700 /million. Any additional coronary artery bypass operations performed will obviously add to the burden on the investigative services.

Increased use of angioplasty will create greater demands on the cardiac laboratories. The impact on the demand for surgery is not clear. Of much more concern, because of the service implications, is the introduction of thrombolytic drugs for the treatment of acute myocardial infarction. These drugs given immediately after infarction may prevent death or limit damage to the myocardium, but such use mav increase survival rates and therefore may add to the pool of patients who will require coronary artery bypass surgery. Many of these patients will require early operation.

The effectiveness of thrombolytic therapy must be assessed; meanwhile it should be available only in centres undertaking this research.

We considered that the UK Cardiac Surgical Register is an excellent professional initiative and a most useful source of data. However, from what has been said earlier a great deal more detail is required-for example, analysis of types of cases operated on for coronary artery disease with mortality and morbidity rates. We were surprised at the paucity of good data about investigative 
activity and medically treated cases. A patient register is essential in order to plan services and to monitor performance in an area of activity that commands such substantial resources and where techniques and results are changing.

(4) What would be the cost and implications for service organisation of increased provisions for investigations and therapy?

In 1982 the numbers of coronary artery bypass operations performed in Britain, per million population, were: Scotland 165; Metropolitan regions 169; rest of the UK 47. The lowest estimate we have accepted of current need is nearly twice the highest and six or seven times the lowest of these figures. To attempt to cost all possible clinical need seemed to us a fruitless exercise. A strong case has been made for coronary artery bypass surgery as the most effective treatment in cases of intractable angina. Therefore, in our view, a clear lead needs to be given nationally about the future direction and rate of expansion of cardiac services so that priority needs are met and the grossly unequal distribution of service across the country is corrected. The achievement of these two goals should be closely monitored. Until the gaps in the data required for future planning are filled we would endorse the suggested rate of 300 coronary artery bypass operations per million, as a realistic short term target, if this represents provision only for high benefit patients.

Taking a notional figure of $\$ 3580$ per operation this would require an additional $£ 35$ million per annum, although some progress towards this figure will have been made by the NHS since 1982.

To arrive at a true assessment of extra cost will require a region by region review to establish: (1) what level of service is currently available and what capacity there is to absorb an expanded workload; the extent to which existing centres are appropriately located; what additional facilities are required by way of theatres, laboratories, and beds (recognising that to introduce additional cardiac surgical work may disturb the balance of services within a district general hospital and may overload other support services including general intensive care facilities); and (2) what additional staff will be required. We have been told that no more than 12 additional cardiac surgeons are required but that the need for additional cardiologists is very much greater if each district general hospital is to have at least one physician with a special interest in cardiology and each main centre up to six. The precise number is difficult to estimate as some appointments will be achieved through changes in workload in related specialties, but a considerable number of nurses may be required for theatres, wards, and intensive care. A separate assessment of the need for investigative facilities at district general hospital level, and of increased facilities for angiography in the regional centres, will need to be made.

Provided regional policy is clear and specific targets are set, we would hope that maximum autonomy and delegated financial responsibility would be given to those with clinical responsibility in the major centres and their associated district general hospitals to develop a service appropriate to local needs. Only in this way can real incentive be given to good practice and efficient performance.

We consider that angioplasty should be fully evaluated in those centres where it is already being developed before it is generally introduced. Also new therapies such as thrombolysis should be introduced only in a limited number of centres until they have been fully evaluated. The proposed national register, which should be centrally funded, should be given priority over any future service development beyond the immediate phase. Attention should also be given to preventive programmes and their evaluation.

These developments require considerable funds. Whether these are found from reallocation of NHS funds or from additional money the problem of assessment of priorities remains. This in turn should take account of estimations of the relative cost effectiveness of other procedures competing for resources. We were impressed by one method of measurement combining quality and duration of life. Further development of this approach is recommended so that it can be of help not only in comparison between coronary artery bypass surgery and other priorities but also between the various subgroups of patients whom it is proposed should be treated by coronary artery bypass surgery. Such techniques would also help to identify health service activities which are being continued despite low benefit.

\section{MEDICINE AND THE MEDIA}

"T OP DOCS BLAST lazy GPs who coin it in" was the title to a short piece in the Sun (31 October, p 5) leaking (inaccurately) one tiny part of the study by Dr David Wilkin and Professor David Metcalfe that we publish today (p 1501). The first sentence (and paragraph) of the Sun scoop continued: "Work shy doctors are only spending 20 hours a week seeing patients a shock report reveals today." The entire piece is constructed around this one inaccurate fact, but the anonymous author doesn't hesitate to include a quote from the "shock report" saying that GPs "spend mornings in the surgery and afternoons on the golf course." The superficial reader will conclude that this quote comes from "the high powered research team"; in fact, it comes from Doctor-for it is the real protagonist of this sorry tale.

Doctor carried this story on the front page of its issue of 1 November under the headline "Report exposes 20-hour week." Recognising the newsworthiness of the "devastating report," the editor sent a proof of the article to the Sun (and to most other national media). The appearance of the Sun report was as much of a shock to Dr Wilkin and Professor Metcalfe as it was to the BMA, who described the report (without having seen it) as "utter rubbish." It was even more of a surprise to Professor John Bain, whose picture appeared with the article and who was quoted in the text, as he had not spoken to Doctor.

Doctor seems to have picked up the story from a meeting in Bath, where Dr Theo Schofield, who knew of the study by Dr Wilkin and
Professor Metcalfe, had off the cuff quoted some of the results. Professor Bain also spoke at this meeting, which is how, he thinks, he came to be quoted in the Doctor article.

Not surprisingly the Sun article attracted much attention from other media, and Professor Metcalfe was besieged by callers. Most of them to their credit (and sadly this doesn't include the Mail on Sunday (4 November, p 8)) backed off when they heard that only one distorted statistic was available and that the full report would be published in the $B M \mathcal{F}$.

One follow up, however, was a leading article in a subsequent issue of Doctor. It attacked the Sun: "One ill informed national newspaper lost no time indulging in a bout of doctor bashing with a grossly distorted interpretation of the study's conclusions. . . ." This seems to be the pot calling the kettle black, but Doctor also had words for Professor Metcalfe and Dr Wilkin. "This is not the first time," it continued, "the public has gained the wrong impression from the bare bones of medical statistics. It is up to Professor Metcalfe and Dr David Wilkin to put some flesh on them...."

Professor Metcalfe and Dr Wilkin must have been infuriated by those two sentences. First they have their results inaccurately leaked, and then they are accused of withholding important information. General practitioners will surely become less and less willing to cooperate with the medical newspapers if they are treated so badly. 